\title{
Treatment of Occipital Neuralgia by Thermal Radiofrequency Ablation
}

\author{
Lance M. Hoffman, MD, ${ }^{1}$ Alaa Abd-Elsayed, MD, MPH, ${ }^{2}$ Tim J. Burroughs, MD, ${ }^{1}$ Harsh Sachdeva, MD ${ }^{1}$ \\ ${ }^{1}$ Department of Anesthesiology, University of Cincinnati, Cincinnati, $\mathrm{OH}^{2}$ Department of Anesthesiology, University of Wisconsin School \\ of Medicine and Public Health, Madison, WI
}

Background: Occipital neuralgia is an uncommon disorder characterized by severe pain involving the posterior scalp in the distribution of the greater and lesser occipital nerves. In cases refractory to pharmacotherapy, invasive treatment options may be warranted. The aim of the present study was to examine the effects of thermal radiofrequency ablation (TRF) on occipital neuralgia. We hypothesized that this procedure would result in long-term pain relief.

Methods: All patients who underwent TRF of the greater and lesser occipital nerves between January 1, 2013 and March 23, 2016 were identified. Medical records were reviewed for preprocedure pain score, 1-month postprocedure pain score, patient-defined percent pain relief according to the visual analog scale (on which zero equals no pain and 10 represents the worst pain imaginable), and length of pain relief. The primary outcomes were the differences between preprocedure and 1month postprocedure pain scores (mean change from baseline), percent pain relief, and patient-reported length of relief.

Results: A total of 50 patients were identified; 4 patients were excluded because of insufficient data as a result of loss to followup. A significant difference was found between preprocedure and postprocedure patient-reported pain scores (6.7 vs 2.7 , respectively; $P<0.001$ ), equating to a mean reduction in pain scores 1 -month postprocedure of $4.0 \pm 3.3$. The mean patientdefined percent pain relief was $76.3 \% \pm 25.0 \%$. The mean patient-reported length of relief was $6.5 \pm 5.1$ months.

Conclusion: This study suggests that TRF may reduce pain scores for approximately 6 months.

Keywords: Denervation, facial neuralgia, facial pain, pulsed radiofrequency treatment

Address correspondence to Lance M. Hoffman, MD, Department of Anesthesiology, University of Cincinnati, $2 F 31$ Albert Sabin Way, PO Box 670531, Cincinnati, OH 45267-0531. Tel: (309) 368-7721. Email: lancemartinhoffman@gmail.com

\section{INTRODUCTION}

Occipital neuralgia is an uncommon disorder characterized by paroxysmal, shooting, or stabbing pain involving the posterior scalp in the distribution of the greater and lesser occipital nerves. The greater and lesser occipital nerves arise from the medial branch of the dorsal ramus of the $\mathrm{C} 2$ spinal nerve. Emerging from below the suboccipital triangle between the obliquus capitis inferior and semispinalis capitis muscles, the greater occipital nerve often pierces the trapezius muscle close to its occipital attachments. At several points along the nerve pathway-from neural foramen to scalp, terminating at the vertex and over the ear-compression, irritation, and friction can contribute to pain. This pain is sometimes associated with dysesthesia or hyperesthesia in the affected area. These symptoms are associated with tenderness to palpation of the affected nerve(s). Responsiveness to local anesthetic blockade of the greater and/or lesser occipital nerves is diagnostic. ${ }^{1}$ Occipital neuralgia is often associated with traumatic head and whiplash-type injuries. ${ }^{2}$ While the incidence is not clearly known because of presumed underreporting and misdiagnosis, Koopman et al estimated that 3.2/100,000 people may suffer from occipital neuralgia. ${ }^{3}$

The diagnosis of occipital neuralgia can be difficult to make, in large part because of the rarity of this disease. The treatment of occipital neuralgia may also pose a challenge for providers because of refractoriness to conservative management. Common first-line treatments often considered for treatment include nonsteroidal antiinflammatory drugs, anticonvulsants, muscle relaxants, massage, and physical therapy. ${ }^{2}$ In cases refractory to pharmacotherapy, invasive treatment options may be indicated. While onabotulinumtoxinA (BOTOX) is commonly used for treatment of chronic migraine and cervical dystonia, insufficient data support its use for the treatment of occipital neuralgia. In such cases, therapeutic occipital nerve blocks with corticosteroids may be warranted. Such nerve blocks provide symptomatic relief and help diagnose a potential target for further treatment. According to recent evidence (2002-2014), however, this type of procedure typically results in temporary relief with a duration of less than 1 month. ${ }^{4-6}$ 
Given the impracticality and deleterious nature of multiple, recurrent steroid injections, treatment strategies with prolonged durations of pain relief have been a point of interest for many pain physicians. Although many interventional and surgical procedures have been described in the literature, few studies have evaluated the efficacy or duration of action of these treatment options..$^{7-9}$ Specifically, published data regarding the utility and efficacy of thermal radiofrequency ablation (TRF) for the treatment of occipital neuralgia are limited. ${ }^{10}$

Given the paucity of published data regarding the utility of TRF for the treatment of occipital neuralgia, this study aimed to delineate the effectiveness of this treatment modality. The overall hypothesis of this study was that TRF would reduce pain for prolonged durations of time.

\section{METHODS}

This retrospective study was approved by the Institutional Review Board of the University of Cincinnati Medical Center (Study ID 2014-1291). Because the study was limited to the review of recorded data, the necessity for obtaining informed consent was waived. All patients who were diagnosed with occipital neuralgia following diagnostic occipital nerve blocks and who subsequently underwent TRF of the greater and lesser occipital nerves at the University of Cincinnati in the Department of Pain Medicine between January 1, 2013 and March 23, 2016 were identified and included in the database.

Of the patients identified, the majority underwent both greater and lesser occipital nerve TRF. To maintain consistency within the sample group, only patients who underwent both greater and lesser occipital nerve TRF were included in this study. Many of the patients identified in this study underwent more than one round of TRF, but only the data from each patient's first TRF procedure were collected. Patients with inadequate data collection because of lack of documentation or loss to follow-up were excluded. All patients who underwent the TRF received nerve blocks for the greater and lesser occipital nerves with or without steroids with the use of $0.25 \%$ bupivacaine. Nerve blocks were performed using anatomic landmarks for both the greater and lesser occipital nerves. Patients who achieved $50 \%$ or more improvement in their pain after the nerve blocks were eligible for TRF.

The TRF procedure was performed in a similar fashion in all patients. Twenty-gauge radiofrequency lesioning $50-\mathrm{mm}$ insulated needles (5- $\mathrm{mm}$ active tip) were placed in proximity to the greater and lesser occipital nerves via the landmarkbased technique. Specifically, the greater occipital nerve was located by palpating the occipital artery, placing the needle just medial to the artery, advancing it perpendicular to the occipital bone until the needle approached the periosteum of the underlying occiput, and redirecting it superiorly as needed until the point of maximum tenderness was identified. The lesser occipital nerve was identified by palpating the mastoid process, placing the second needle immediately medial to the mastoid process, and advancing it superiorly until the maximum point of tenderness was identified. Following placement of the needles, sensory stimulation at $50 \mathrm{~Hz}$ was used to confirm proximity to the underlying greater and lesser occipital nerves by reproducing pain or paresthesia in the area of interest. TRF was carried out in lesion mode after injecting $1-2 \mathrm{~mL}$ of $2 \%$ lidocaine. The settings were $80^{\circ} \mathrm{C}$ and 180 seconds.

We reviewed medical records for patient demographic data such as age and sex, disease laterality, preprocedure and postprocedure pain scores, patient-defined percent pain relief, and patient-defined length of relief. The preprocedure pain score was defined as the visual analog scale (VAS) score the patient reported for the pain originating in the occipital head during the office visit prior to undergoing the procedure. The VAS is scored from 0 (no pain) to 10 (worst pain imaginable). The postprocedure pain score was defined as the VAS score the patient reported at the 1-month postprocedure office visit when the patient was asked specifically about posterior headache and pain radiating from the posterior head. The primary outcomes were the difference between the preprocedure and 1-month postprocedure VAS scores (mean change from baseline), percent pain relief (as defined by the patient's subjective impression), and patient-reported length of relief of occipital head pain. Continuous variables were compared using paired $t$ tests assuming equal or unequal variance when appropriate. For clarity, unequal variance was only used when comparing males and females. Regression analysis was used to interpret any correlation between patient-reported duration of pain relief and patient-perceived pain relief at the 1month postprocedure visit. Two-tailed $P$ values $\leq 0.05$ were considered to be statistically significant.

\section{RESULTS}

We identified 50 patients who had been diagnosed with occipital neuralgia following diagnostic occipital nerve blocks and who subsequently underwent TRF of the greater and lesser occipital nerves between January 1, 2013 and March 23, 2016. Four patients were excluded because of insufficient data, so 46 patients were included in the analysis. The average patient age was $46.5 \pm 13.6$ years, and the majority of patients were female ( $82.6 \%$ female vs $17.4 \%$ male). In this patient population, $54.3 \%$ were diagnosed with bilateral occipital neuralgia, and $45.7 \%$ had unilateral occipital neuralgia. These patients underwent bilateral TRF and unilateral TRF, respectively.

We found a significant difference between preprocedure and 1-month postprocedure patient-reported VAS scores (6.7 vs 2.7, $P<0.001$ ), a mean reduction of $4.0 \pm 3.3$ (Figure 1). As depicted in Figure 2, the patient-reported length of relief following TRF varied among patients, but the mean was $6.5 \pm 5.1$ months. This mean was calculated from the data for 39 patients. Results from the remaining 7 patients were excluded because the clinical documentation failed to specifically define the length of relief. In the majority of these cases, the clinical notation did not clearly define the length of relief because of the lack of ongoing follow-up beyond 1 month.

Based on data from 44 patients, the mean percent of pain relief reported by patients at the 1-month follow-up visit was $76.3 \% \pm 25.0 \%$ (Figure 3 ). Clinical documentation for the other 2 patients defined their pain relief as "significant," which could not be represented in a numerical fashion.

Regression analysis to identify any correlating trend between patient-reported duration of relief and patientperceived percent relief at the 1-month postprocedure office visit showed a statistically significant trend (Figure 4) despite 


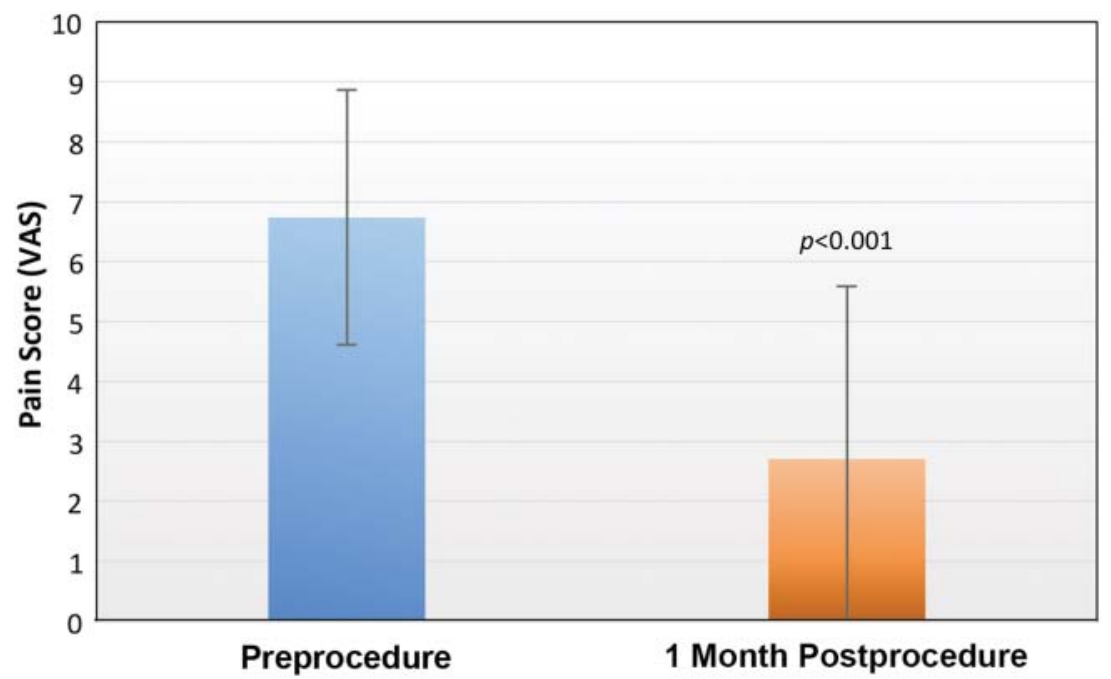

Figure 1. Mean patient-reported pain scores preprocedure and 1 month after thermal radiofrequency ablation treatment $(n=46)$ as measured with the visual analog scale (VAS) in which zero corresponds to no pain and 10 corresponds to the worst pain imaginable.

high variability within the data set $\left(R^{2}=0.148\right.$; adjusted $\left.R^{2}=0.125 ; P=0.016\right)$. No statistically significant differences were identified between males and females when comparing patient-reported pain scores, percent relief, or length of relief $(P=0.122, P=0.475$, and $P=0.490$, respectively).

\section{DISCUSSION}

Investigators have studied the use of pulsed radiofrequency (PRF), a nondestructive form of radiofrequency therapy for the treatment of occipital neuralgia. Several studies on occipital nerve PRF have shown statistically significant improvements in pain scores and quality of life and reductions in pain medication use. ${ }^{8,9,11}$ Cohen et al compared the effects of PRF to occipital nerve blocks with steroids in a randomized, double-blind study and found PRF to be superior in terms of average pain score reductions at 6 weeks through 6 months. ${ }^{9}$ Given these findings, Cohen et al sug- gest that PRF should be considered as a treatment modality for prolonged benefit beyond occipital nerve blocks utilizing steroids.

Depending on how radiofrequency energy is applied to neural tissues, radiofrequency therapy can be defined as thermal or pulsed. TRF produces more heat that results in neural disruption via the creation of a destructive lesion. PRF, in comparison, delivers short bursts of high-amplitude current between comparatively long pauses that allow for the dissipation of heat. ${ }^{12}$ Neural tissue is preserved by allowing this heat to dissipate as proven in histologic studies. ${ }^{13-16}$ The prevailing theory regarding the mechanism of action of PRF is related to the microscopic damage incurred on axonal microtubules and microfilaments. These microscopic changes are thought to result in alterations in synaptic transmission. $^{12-17}$ Of particular interest, the effects of PRF appear to be most pronounced in C-fibers compared to A-

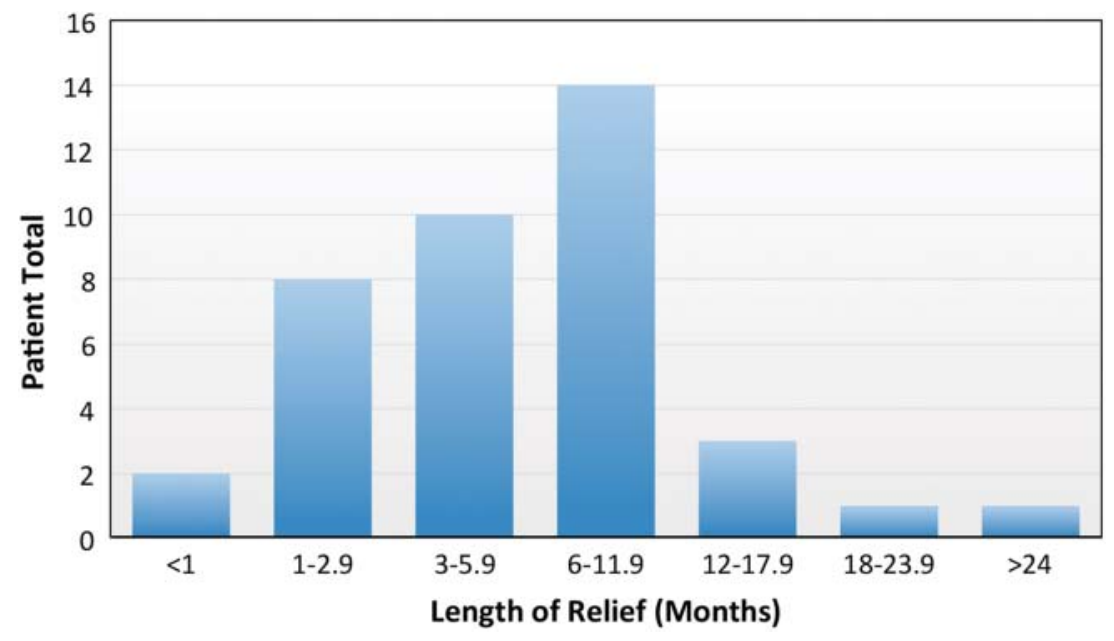

Figure 2. Patient-defined duration of pain relief in months following thermal radiofrequency ablation $(n=39)$. 


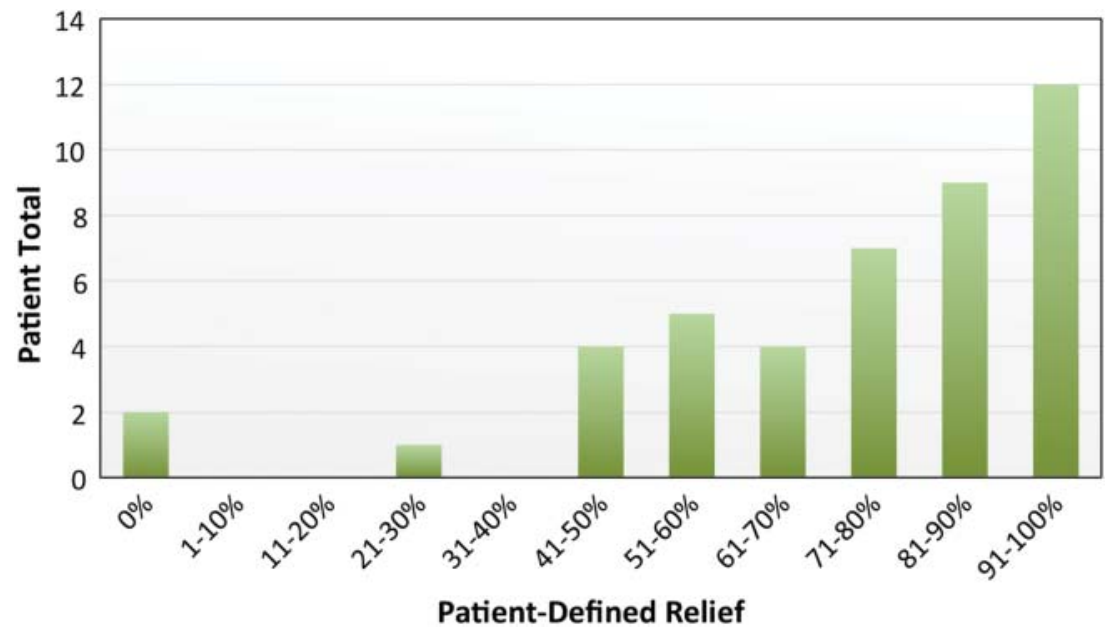

Figure 3. Patient-defined percent improvement in pain relief from baseline following thermal radiofrequency ablation $(n=44)$.

delta and A-beta fibers. ${ }^{16}$ Despite the theoretical benefits of having an improved margin of safety with PRF, inadequate clinical evidence supports the use of PRF as a comparable alternative to TRF for the treatment of chronic pain conditions. ${ }^{12,18}$

Although no studies have specifically compared the outcomes of TRF vs PRF for the treatment of occipital neuralgia, studies have compared these two radiofrequency modalities for trigeminal neuralgia, a similar neuropathic type-mediated headache disorder. In a prospective, randomized, double-blind study by Erdine et al, TRF of the Gasserian ganglion exhibited superior efficacy compared to PRF for the treatment of idiopathic trigeminal neuralgia. ${ }^{19} \mathrm{~A}$ total of 40 patients were allocated to either the TRF group or the PRF group. While the TRF group had a statistically significant reduction in pain scores and improvement in patient satisfaction, only $10 \%$ (2 of 20 ) of patients in the PRF group reported decreased pain scores. Patients in the PRF group subsequently underwent TRF after 3 months because of continued, intractable pain. Following TRF, the PRF group also showed statistically significant reductions in pain in addition to improvement in satisfaction, suggesting the superiority of TRF compared to PRF. ${ }^{19}$

Our study results are in agreement with another study in which TRF applied to pericranial nerves improved pain in patients with headache conditions. ${ }^{20}$ These findings indicate that TRF may be an effective treatment option for occipital neuralgia and should be considered for patients with occipital neuralgia who do not respond to pharmacologic therapy or cannot tolerate it. In our experience, TRF is a relatively low-risk option for headache management; it has no medication side effects and a low incidence of complications.

Other minimally invasive procedures may be useful in the treatment of occipital headache, such as block or ablation of upper cervical medial branches for facet-mediated pain causing cervicogenic headache. However, as occipital nerve blocks are arguably lower risk than C2 medial branch blocks and can be performed reliably without image guidance, they

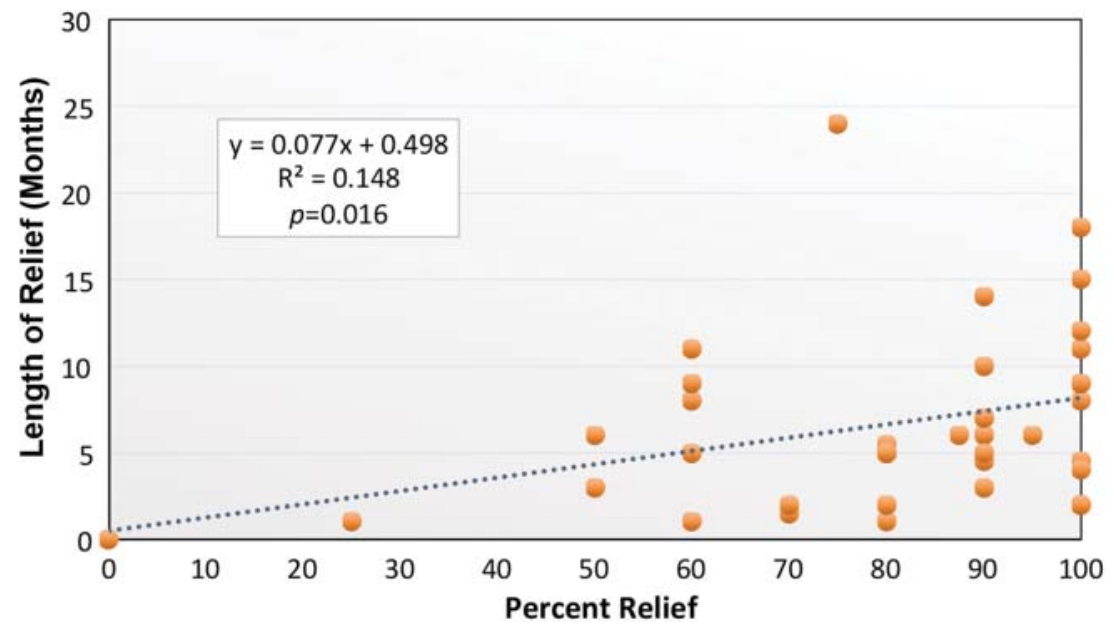

Figure 4. Regression analysis indicated a correlation between patient-reported pain relief duration and the patient-reported percent pain relief at the 1month postprocedure office visit $(n=39)$. 
often serve as part of the initial diagnostic algorithm for determining the most efficacious therapeutic target. Given the low observed prevalence of occipital neuralgia, a low-risk method for diagnosing and treating this debilitating condition could eliminate unnecessary treatments with increased side effects and complications.

As stated earlier, a retrospective review of the general population in the Netherlands reported an incidence of occipital neuralgia of 3.2 per 100,000 people, but this study likely underestimated the frequency of this disorder because of underreporting. ${ }^{3}$ Among the most likely reasons for underreporting is a lack of awareness regarding occipital neuralgia among primary care physicians who participated in the data collection. Additionally, the primary care physicians who created this database were not seeking to diagnose and treat specific facial pain conditions. The study, as a result, only reported cases of occipital neuralgia in which the complaint was precisely addressed. As a result, the epidemiologic data of this disorder have yet to be elucidated.

Limitations of our study include those commonly associated with retrospective studies, most notably, researcher bias and confounding variables. Expectations for a particular outcome can influence the interpretation of a procedure result. A more extensive questionnaire including functional change may be used in future prospective studies to create a more reliable measure of outcome. In addition, no concurrent therapies were accounted for before, during, or following treatment with TRF. Conceivably, such therapies could affect the results attributed to the procedure.

Another potential limitation of this study is the use of a landmark-based technique for identification of the greater and lesser occipital nerves. Although superiority has yet to be established regarding the use of an ultrasound-guided technique over the landmark-based technique for this procedure, recent literature has typically favored the use of imageguided techniques. ${ }^{21}$ Because the general consensus among most interventionists is that ultrasound guidance provides improved accuracy in needle placement and safety (with regard to avoidance of vascular structures), the results in this study may be negatively impacted by the method used for needle placement because of an inherent lack of accuracy.

\section{CONCLUSION}

This study demonstrated a statistically significant reduction in patient-reported pain scores following TRF with an average patient-reported length of relief greater than 6 months. These results add to the evidence supporting the use of TRF as a treatment modality for patients diagnosed with occipital neuralgia who have failed conservative therapies. However, given the inherent limitations of retrospectives studies, a prospective, randomized controlled trial should be undertaken to elucidate the effectiveness of TRF for the treatment of occipital neuralgia. Additionally, given the high variability in duration of pain relief reported by patients in this study, further studies may also help delineate patient factors that play a role in the efficacy of this procedure.

\section{ACKNOWLEDGMENTS}

The authors gratefully acknowledge Dr James Fortman II, Dr Jim Bruns, and Denise Richardson for their assistance in support of this project. Dr Abd-Elsayed is a consultant for Medtronic, Halyard, Axsome, Ultimaxx Health, Innocol, and SpineLoop. The other authors have no financial or proprietary interest in the subject matter of this article.

\section{REFERENCES}

1. Headache Classification Committee of the International Headache Society (HIS). The international classification of headache disorders, 3rd edition (beta version). Cephalalgia. 2013 Jul;33(9):629-808. doi: 10.1177/0333102413485658.

2. Dougherty C. Occipital neuralgia. Curr Pain Headache Rep. 2014 May;18(5):411. doi: 10.1007/s11916-014-0411-x.

3. Koopman JS, Dieleman JP, Huygen FJ, de Mos M, Martin CG, Sturkenboom MC. Incidence of facial pain in the general population. Pain. 2009 Dec 15;147(1-3):122-127. doi: 10.1016/j. pain.2009.08.023.

4. Afridi SK, Shields KG, Bhola R, Goadsby PJ. Greater occipital nerve injection in primary headache syndromes-prolonged effects from a single injection. Pain. 2006 May;122(1-2):126-129.

5. Lambru G, Abu Bakar N, Stahlhut L, et al. Greater occipital nerve blocks in chronic cluster headache: a prospective openlabel study. Eur J Neurol. 2014 Feb;21(2):338-343. doi: 10.1111/ ene.12321.

6. Peres MF, Stiles MA, Siow HC, Rozen TD, Young WB, Silberstein SD. Greater occipital nerve blockade for cluster headache. Cephalalgia. 2002 Sep;22(7):520-522.

7. Vu T, Chhatre A. Cooled radiofrequency ablation for bilateral greater occipital neuralgia. Case Rep Neurol Med. 2014;2014:257373. doi: 10.1155/2014/257373.

8. Vanelderen $P$, Rouwette $T$, De Vooght $P$, et al. Pulsed radiofrequency for the treatment of occipital neuralgia : a prospective study with 6 months of follow-up. Reg Anesth Pain Med. 2010 Mar-Apr;35(2):148-151.

9. Cohen SP, Peterlin BL, Fulton L, et al. Randomized, doubleblind, comparative-effectiveness study comparing pulsed radiofrequency to steroid injections for occipital neuralgia or migraine with occipital nerve tenderness. Pain. 2015 Dec;156 (12):2585-2594. doi: 10.1097/j.pain.0000000000000373.

10. Waldman SD. Atlas of Interventional Pain Management. 4th ed. Philadelphia, PA: Elsevier Saunders, 2015.

11. Huang JH, Galvagno SM Jr, Hameed H, et al. Occipital nerve pulsed radiofrequency treatment: a multi-center study evaluating predictors of outcome. Pain Med. 2012 Apr;13 (4):489-497. doi: 10.1111/j.1526-4637.2012.01348.x.

12. Chua NH, Vissers KC, Sluijter ME. Pulsed radiofrequency treatment in interventional pain management: mechanisms and potential indications-a review. Acta Neurochir (Wien). 2011 Apr;153(4):763-771. doi: 10.1007/s00701-010-0881-5.

13. Bogduk N. Pulsed radiofrequency. Pain Med. 2006 Sep-Oct; 7 (5):396-407.

14. Vallejo R, Tilley DM, Williams J, Labak S, Aliaga L, Benyamin RM. Pulsed radiofrequency modulates pain regulatory gene expression along the nociceptive pathway. Pain Physician. 2013 Sep-Oct;16(5):E601-E613.

15. Erdine S, Yucel A, Cimen A, Aydin S, Sav A, Bilir A. Effects of pulsed versus conventional radiofrequency current on rabbit dorsal root ganglion morphology. Eur J Pain. 2005 Jun;9(3):251-256.

16. Erdine S, Bilir A, Cosman ER, Cosman ER Jr. Ultrastructural changes in axons following exposure to pulsed radiofrequency fields. Pain Pract. 2009 Nov-Dec;9(6):407-417. doi: 10.1111/ j.1533-2500.2009.00317.x.

17. Emril DR, Ho KY. Treatment of trigeminal neuralgia: role of radiofrequency ablation. J Pain Res. 2010 Dec 12;3:249-254. doi: 10.2147/JPR.S14455. 
18. van Boxem $K$, van Eerd $M$, Brinkhuizen T, Patijn J, van Kleef $M$, van Zundert J. Radiofrequency and pulsed radiofrequency treatment of chronic pain syndromes: the available evidence. Pain Pract. 2008 Sep-Oct;8(5):385-393. doi: 10.1111/j.15332500.2008.00227.x.

19. Erdine S, Ozyalcin NS, Cimen A, Celik M, Talu GK, Disci R. Comparison of pulsed radiofrequency with conventional radiofrequency in the treatment of idiopathic trigeminal neuralgia. Eur J Pain. 2007 Apr;11(3):309-313.
20. Abd-Elsayed A, Kreuger L, Wheeler S, Robillard J, Seeger S, Dulli $D$. Radiofrequency ablation of pericranial nerves for treating headache conditions: a promising option for patients. Ochsner J. 2018 Spring;18(1):59-62.

21. Vanderhoek MD, Hoang HT, Goff B. Ultrasound-guided greater occipital nerve blocks and pulsed radiofrequency ablation for diagnosis and treatment of occipital neuralgia. Anesth Pain Med. 2013 Sep;3(2):256-259. doi: 10.5812/aapm.10985.

This article meets the Accreditation Council for Graduate Medical Education and the American Board of Medical Specialties Maintenance of Certification competencies for Patient Care and Medical Knowledge. 